\title{
Effectiveness of the Premarital Education Programme in Iran
}

\author{
Mahdieh Yazdanpanah, ${ }^{1}$ Mohammad Eslami, ${ }^{1}$ and Nouzar Nakhaee ${ }^{2}$ \\ ${ }^{1}$ Department of Population and Family Health, Ministry of Health and Medical Education, Tehran, Iran \\ ${ }^{2}$ Neuroscience Research Center, Institute of Neuropharmacology, Kerman University of Medical Sciences, \\ P.O. Box 76175-113, Kerman, Iran
}

Correspondence should be addressed to Nouzar Nakhaee; nakhaeen@yahoo.com

Received 8 January 2014; Accepted 5 February 2014; Published 6 March 2014

Academic Editors: J. Konde-Lule and A. Zaborskis

Copyright (C) 2014 Mahdieh Yazdanpanah et al. This is an open access article distributed under the Creative Commons Attribution License, which permits unrestricted use, distribution, and reproduction in any medium, provided the original work is properly cited.

\begin{abstract}
Introduction. Iran is confronted with increasing trend of divorce in recent decades. Considering the probable role of sexual dissatisfaction in the breakdown of marriages, the Ministry of Health recently decided to enrich the educational content of the premarital education programmes. The aim of this study was to compare the effectiveness of the new program with the classic one. Methods. Three hundred fifty-one women were divided into two premarital education groups: classic education $(n=162)$ and new education $(n=189)$. The mental health, quality of life, marital satisfaction, and sexual satisfaction of women were evaluated one year after marriage. Results. At one-year followup, both groups showed improvement in mental health $(P<0.001)$; however physical health-related quality of life decreased in both groups. The two groups showed no significant difference in terms of sexual satisfaction and marital satisfaction. Marital satisfaction showed significant correlation with sexual satisfaction $(r=0.55, P<0.001)$. Conclusion. The new program of premarital education showed no superiority over the classic method. Considering the increasing rate of divorce in Iran, the premarital education programmes may not be successful in achieving their goals. Revision of the premarital education programme is warranted.
\end{abstract}

\section{Introduction}

As the backbone of society, marriage is considered to be one of the most important institutions in the world [1]. In most societies, be it in the East or West, most people marry at some stage during their life $[1,2]$. There are a variety of motives behind marriage, among them being the need for friendship, social and emotional support, love, and sex [3]. Of course, it should be considered that in marriage, unlike in cohabitation, emotional satisfaction with sex is important due to the couples' commitment toward each other [3]. However, everybody, regardless of his/her motives for marriage, expects a healthier and happier life after marriage.

More recently, divorce and marital instability are an increasing trend in Iran; while most countries with the highest divorce rates are in the West, Iran is also considered a highdivorce country [4]. In 2011, the rate of divorce in Iran was 2.5 per 1000 people, which is even higher than the divorce rates in some western countries such as Ireland, Italy, and Spain. In Tehran, the capital of Iran, more than 30\% of marriages end in divorce [4]. There are different reasons for the increasing divorce rates in Iran, including rapid socioeconomic changes resulting from modernization and urbanization, women's empowerment, and a decreasing stigma associated with divorce [5]. One of the factors playing a role in divorce is sexual dissatisfaction [6]. Many researchers have emphasized the role of sexual satisfaction in causing marital fulfillment $[6,7]$. A bidirectional relationship can be imagined between sexual and marital satisfaction [8]; most longitudinal researchers have come to the conclusion that sexual satisfaction predicts marital satisfaction [9-11].

In spite of several existing studies that focus on the role of sexuality in the continuity of common life, marital sexuality continues to be the most neglected subject in the sex research literature [7].

In Iran, the results of studies show a significant relationship between sexuality and marital satisfaction $[12,13]$. Further, sexual dissatisfaction has been mentioned as one of 
the reasons for divorce. According to an Iranian report, $41.4 \%$ of divorced women were unhappy with their sex life and $74 \%$ of divorced men were sexually dissatisfied [2]. On the other hand, it has been postulated that holding sex skills training classes for married women can increase marital satisfaction [14]. As sex is considered to be a taboo subject in Iran, there is no sex education in schools, which means that premarital education classes are the only formal opportunity for presenting sexual education. These classes are held in compact sessions before marriage and the certificate for passing these classes is one of the legal requirements of marriage. This educational programme has been running since 1993 [15].

The premarital period is one of the critical points for the prevention of serious relationship problems. In many developed countries like England and the United Sates, couples are encouraged to participate in premarital counselling programmes. The effectiveness of these programmes in increasing marital satisfaction has been proven in both developed countries [16] and in countries with a similar culture to Iran [17].

With respect to the increasing divorce rate in Iran and the probable role played by sexual dissatisfaction in the breakdown of marriages, the Ministry of Health recently aimed to improve the educational content of the premarital education programmes. Consequently, sexual education is presented more comprehensively in the format of both lectures and videos. Due to the lack of research regarding the impact of premarital education programmes in Iran, this study attempts to compare the effectiveness of the new educational programme with the classic one. In this study, outcome variables are mental health, quality of life, and marital satisfaction after one year of marriage. Moreover, the relationship between marital satisfaction and sexual satisfaction, quality of life, and mental health was evaluated.

\section{Methods}

2.1. Setting and Participants. This true experimental study was performed in the premarital education centre in Kerman, Iran. We calculated that 180 subjects would be required for the study to provide over $80 \%$ power to detect a $10 \%$ difference in the marital satisfaction score. Although the educational programme is held for both men and women separately, only women were included in this study because in Iranian culture it is more convenient to ask women about sexual issues than men. Moreover, based on our previous experience, women are generally more available for follow-up research due to the fact that they are less busy than men. The aim of the study was explained to participants before they were randomly allocated to one of the two groups: classic or new education. In regard to participant consent, all participants were asked to fill out a questionnaire which was included as part of the study. Participants were of course allowed to exit the study during any phase of the research. The Ethical Committee of Kerman University of Medical Sciences has approved this research (approval code: K/90/226).

In the control group, the classic method of premarital education was applied. The educational classes included 25 women; a female lecturer presented a 90 -minute presentation about the genital organs of males and females, menstruation, pregnancy, and family planning.

In the intervention group, some educational aid instruments (posters and moulage) were used to supplement the lecture. An educational video was also presented to participants. This film covered a range of issues including emotional relations of marital life from the perspective of a psychologist, issues about Islam and the foundations of family from the perspective of a religious expert, and the anatomy of male and female genital organs, the three processes related to fertility (menstruation, pregnancy, and abortion) as well as sexual relations during early marital life from the perspective of a trained teacher. Further, different family planning methods were also covered during the training. This educational course lasted for 2 hours; the trainers had previously passed a 3-day training workshop totalling 24 hours of tuition. Therefore, this new method, in addition to being applied in a different way, was richer in content. In addition, an educational book was given to the participants of this group.

2.1.1. Measurement Tools. Before holding educational classes, participants were asked to complete an SF-12 questionnaire for the purpose of evaluating their quality of life and a GHQ12 questionnaire to evaluate their mental health. The SF-12 questionnaire included two scales of physical and mental health composite scores (PCS and MCS) which evaluated participant's mental and physical functions. These two scales evaluate the overall health-related quality of life in a score ranging from 0 to 100 . The higher score shows better quality of life [18]. GHQ-12 included 12 questions for evaluating mental health with a lower score showing better mental health [19]. The reliability and validity of the Persian version of both questionnaires were approved. Cronbach's alpha for GHQ12 and SF-12 in our study was computed to be 0.74 and 0.82 , respectively. One year after marriage, participants were asked to complete these two questionnaires again in addition to the sexual satisfaction inventory [20] and the ENRICH Marital Satisfaction Scale [21]. The short form of this scale was used in the final analysis. The reliability and validity of the Brief version were approved in the Iranian population [22]. Sexual satisfaction consisting of sexual attitude (2 questions) and sexual practice (4 questions) was defined by 6 questions from Bahrami et al. [20]. The following question was also asked to explore the perceived effectiveness of the two educational programmes from the viewpoint of participants: "in your opinion, how effective has the class been in improving your marital relationships?"

The scores ranged from a minimum of zero (completely ineffective) to a maximum of 10 (completely effective). A $500000 \mathrm{R}$ gift card was given to each participant who completed the second phase of the questionnaire.

To compare the scores of two groups, $t$-test was applied. The scores of quality of life and mental health before and after the intervention were compared using paired $t$-test. ANCOVA was used to compare the scores of questionnaires between the two groups one year after the intervention. To compare categorical variables between the two groups, Chisquare test was used. Pearson coefficient of correlation was 
TABLE 1: Demographic variables in intervention and control groups.

\begin{tabular}{|c|c|c|c|}
\hline Characteristics & Classic group $(n=162)$ & Experimental group $(n=189)$ & $P$ value \\
\hline Women's age (mean $\pm \mathrm{SE})$ & $23.4 \pm 0.4$ & $23.8 \pm 0.4$ & 0.421 \\
\hline Men's age $($ mean $\pm \mathrm{SE})$ & $26.8 \pm 0.4$ & $27.2 \pm 0.4$ & 0.580 \\
\hline Duration of knowing each other before marriage (months) & $13.0 \pm 1.1$ & $13.0 \pm 1.0$ & 0.991 \\
\hline \multicolumn{4}{|l|}{ Women's education } \\
\hline$\leq$ Elementary & $5(3.1)$ & $6(3.2)$ & \multirow{4}{*}{0.169} \\
\hline Secondary & $23(14.2)$ & $17(9.0)$ & \\
\hline High school diploma & $61(37.7)$ & $84(44.4)$ & \\
\hline College & $73(45.1)$ & $82(43.4)$ & \\
\hline \multicolumn{4}{|l|}{ Men's education } \\
\hline$\leq$ Elementary & $6(3.7)$ & $10(5.3)$ & \multirow{4}{*}{0.366} \\
\hline Secondary & $33(20.4)$ & $39(20.7)$ & \\
\hline High school diploma & $66(40.7)$ & $86(45.7)$ & \\
\hline College & $57(35.2)$ & $53(28.2)$ & \\
\hline \multicolumn{4}{|l|}{ Perceived income adequacy } \\
\hline Adequate & $151(95.2)$ & $178(94.2)$ & \multirow{2}{*}{0.708} \\
\hline Inadequate & $11(6.8)$ & $11(5.8)$ & \\
\hline \multicolumn{4}{|l|}{ Consanguineous marriage } \\
\hline Yes & $50(30.9)$ & $63(33.3)$ & \multirow{2}{*}{0.622} \\
\hline No & $112(69.1)$ & $126(66.7)$ & \\
\hline
\end{tabular}

TABLE 2: Comparison of sexual attitudes and practices (number of "yes" answers) between the two groups.

\begin{tabular}{|c|c|c|c|c|}
\hline & Item & $\begin{array}{l}\text { Classic group } \\
\quad(n=162)\end{array}$ & $\begin{array}{c}\text { Experimental } \\
\text { group } \\
(n=189) \\
\end{array}$ & $P$ value \\
\hline \multirow[t]{2}{*}{ Attitude } & $\begin{array}{l}\text { In my belief, sexual relationships are very important } \\
\text { and interesting in marital life }\end{array}$ & $161(99.4)$ & $189(100)$ & 0.462 \\
\hline & Sexual relationships improve emotional relationships & $157(96.9)$ & $183(96.8)$ & 0.962 \\
\hline \multirow{4}{*}{ Practice } & My husband does not satisfy me sexually & $15(9.3)$ & $21(11.1)$ & 0.569 \\
\hline & My husband is too rough during sexual intercourse & $8(4.9)$ & $22(11.6)$ & 0.400 \\
\hline & Our sexual relationship is too hasty & $14(8.6)$ & $22(11.6)$ & 0.356 \\
\hline & I try to avoid sexual relationship with my husband & $13(8.0)$ & $9.0(4.8)$ & 0.209 \\
\hline
\end{tabular}

used to determine the relationship between the quality of life and mental health scores with sexual and marital satisfaction.

\section{Results}

From 183 participants in the classic education group 21 participants and from 203 participants in the new education group 14 did not participate in the second phase of the study (one-year followup). No difference in underlying variables was observed between these participants and others who remained in the study $(P>0.05)$. Demographic features of the two studied groups are presented in Table 1; it is observed that there was no significant difference between the two groups in regard to these variables $(P>0.05)$.

Only $0.3 \%$ of participants disagreed with the fact that sexual relationships are very important and interesting in marital life and $97 \%$ believed that sexual relationships improve emotional relationships (Table 2). On the whole, the responses regarding sexual satisfaction were similar between the two groups (Table 2).

In both groups, the score of mental health-related quality of life showed significant improvement after one year of marriage compared to those before marriage, whilst SF-12 summary scales of physical health deteriorated after one year of marriage (Table 3).

No significant difference was found between the two groups in regard to the scores of mental health, quality of life, marital satisfaction, and sexual practice after one year of marriage $(P>0.05)$.

Divorce rate after one year of common life was $3.3 \%$ in the classic education group compared to $3.4 \%$ in the new education group $(P=0.972)$.

The effectiveness of educational classes in improving marital relationships was more from the viewpoint of the women in the new education group compared to the women in the classic education group (perceived impact scores were,resp., $8.0 \pm 0.2$ and $7.3 \pm 0.2, P<0.05)$. 
TABLE 3: Scores (mean \pm SE) of both mental health and quality of life before and after one year of marriage.

\begin{tabular}{|c|c|c|c|c|}
\hline Group & Variable & Before marriage & One year after marriage & $P$ value \\
\hline \multirow{4}{*}{ Classic group $(n=162)$} & Mental health & $5.09 \pm 0.22$ & $2.77 \pm 0.24$ & $<0.001$ \\
\hline & Mental health-related quality of life & $66.28 \pm 1.38$ & $68.26 \pm 1.38$ & 0.159 \\
\hline & Physical health-related quality of life & $74.02 \pm 1.38$ & $68.39 \pm 1.53$ & $<0.001$ \\
\hline & Overall quality of life & $70.16 \pm 1.20$ & $68.32 \pm 1.28$ & 0.132 \\
\hline \multirow{4}{*}{ Experimental group $(n=189)$} & Mental health & $4.90 \pm 0.20$ & $2.74 \pm 0.21$ & $<0.001$ \\
\hline & Mental health-related quality of life & $66.84 \pm 1.27$ & $68.25 \pm 1.25$ & 0.350 \\
\hline & Physical health-related quality of life & $75.22 \pm 1.10$ & $67.54 \pm 1.26$ & $<0.001$ \\
\hline & Overall quality of life & $71.03 \pm 0.99$ & $67.90 \pm 1.07$ & 0.007 \\
\hline
\end{tabular}

Marital satisfaction showed a significant correlation with mental health scores after marriage $(r=0.51, P<0.00)$, quality of life after marriage $(r=0.42, P<0.001)$, and sexual satisfaction $(r=0.55, P<0.001)$.

\section{Discussion}

Although the effectiveness of premarital education in the improvement of marital relationships, at least in the short term, has been proven in developed countries [16, 23], the effectiveness of these programmes in developing countries has not been adequately studied. The present study highlights that participants from both study groups experienced improvements to both their mental health and mental healthrelated quality of life after one year of marriage; however the score regarding physical health-related quality of life decreased. Sexual satisfaction and marital satisfaction one year after marriage were comparable in the two studied groups.

The advantage of this study was using a true experimental design with a large sample size [23], while its main disadvantage was the lack of a no-intervention group. The reason for not considering a no-intervention group was due to the Ethical Committee disagreeing over the idea of depriving one group from receiving the standard educational programme.

This study compared the effectiveness of delivering premarital education through a lecture only (with less emphasis on sexual education) with the effectiveness of the programme using a combination of lecture, educational video, and book (with more emphasis on sexual education). The two groups were comparable in regard to the underlying variables. The major aspect of the educational content in both groups was related to sexual issues. Sexual satisfaction scores of the two groups showed no significant difference in the oneyear followup. Nearly $100 \%$ of participants admitted the importance of sexual relationships in marital life and believed that sexual relationships improve emotional relationships (Table 2). In other studies, couples have also considered sexual satisfaction as one of the most important components of marital happiness and functioning [24]. Since marital satisfaction is a subjective feeling, the perceived importance of sexual relationships in providing marital happiness from women's viewpoint could be evidence of the importance of premarital sex education. However, in the present study, similar to other studies, women believed that sexual satisfaction plays an essential role [9]. The correlation between marital satisfaction and sexual satisfaction was high $(r=$ $0.55)$. In previous studies undertaken in Iran [2] and in the United States [7], the correlation coefficient between these two constructs was almost 0.5. Mental health in both groups showed significant improvement after one year of marriage compared to before marriage (Table 3 ). Longitudinal studies have shown improvement in emotional well-being and mental health outcomes and an increase in overall happiness after marriage [3]. Since this study lacked a no-intervention group, we cannot determine the exact role of educational programmes in the improvement of mental health. PCS that show physical health-related quality of life, as opposed to MCS, decreased one year after marriage. PCS include aspects such as "physical functioning," "role limitations due to physical problems," and "bodily pain." Therefore, it seems that women's physical health declines after one year of marriage when compared to their physical health before marriage. This might be attributed to the fact that in Iran women and girls are not expected to do heavy household tasks when they are single, but after marriage household tasks are women's responsibility even if they work outside of the home [25].

This study highlights that marital satisfaction has a significant correlation with mental health scores and quality of life after marriage. In other studies, marital satisfaction also correlated with mental health [7] and quality of life [25]; however the relationship was reciprocal and how much each played a role in causing the other remains controversial [25].

There was no significant difference between the two groups in regard to sexual and marital satisfaction. That is, in spite of enriching the educational content and adding additional educational tools to supplement a lecture, no significant improvement was observed in terms of outcomes. After one year, the divorce rate between the two groups was similar (one in each 30 couples). Although subjectively the new education group believed in the effectiveness of premarital educational programmes in improving marital relationships more than the control group $(P<0.05)$, objectively there was no significant difference between the two groups. Although the classic premarital educational programme has been implemented in Iran for the past 20 years and participating in these classes has been compulsory for all marriage volunteers, the divorce rate has increased over the last two decades. In other words, the long-term 
effectiveness of this programme is under question. Likewise, in developed countries, although premarital education and counselling have been effective in the short term, the longterm effectiveness of these interventions is questionable and has not been fully supported by research. Therefore, it is necessary that this programme be evaluated in terms of the method of implementation and educational content. The low rate of divorce per cent in comparison to the country's divorce rate is due to following up subjects for only a one-year period, as the divorce rate is lower in the first postmarital year than during the subsequent 5-year period [4].

\section{Conclusion}

Overall, sexual satisfaction, marital satisfaction, and divorce rate showed no difference between the two educational methods. Since the effectiveness of premarital programmes in increasing marital satisfaction has been well established in different countries and considering the increasing trend of divorce in Iran, we can conclude that there is no convincing evidence that the premarital education programmes are efficacious in terms of marital satisfaction. Therefore, policymakers need to pay more attention to the new premarital educational programme to find the pitfalls of the programme.

\section{Conflict of Interests}

The authors declare that they have no conflict of interests.

\section{References}

[1] J. E. Myers, J. Madathil, and L. R. Tingle, "Marriage satisfaction and wellness in India and the United States: a preliminary comparison of arranged marriages and marriages of choice," Journal of Counseling and Development, vol. 83, no. 2, pp. 183190, 2005.

[2] A. Rahmani, E. M. Khoei, and L. A. Gholi, "Sexual satisfaction and its relation to marital happiness in Iranians," Iranian Journal of Public Health, vol. 38, no. 4, pp. 77-82, 2009.

[3] L. J. Waite and E. L. Lehrer, "The benefits from marriage and religion in the United States: a comparative analysis," Population and Development Review, vol. 29, no. 2, pp. 255-276, 2003.

[4] A. Aghajanian and V. Thompson, "Recent divorce trend in Iran," Journal of Marriage and Family, vol. 75, no. 2, pp. 342-362, 2013.

[5] M. J. Abbasi-Shavazi, A. Askari-Nodoushan, and A. Thornton, "Family life and developmental idealism in Yazd, Iran," Demographic Research, vol. 26, pp. 207-238, 2012.

[6] F. S. Christopher and S. Sprecher, "Sexuality in marriage, dating, and other relationships: a decade review," Journal of Marriage and Family, vol. 62, no. 4, pp. 999-1017, 2000.

[7] S. Litzinger and K. C. Gordon, "Exploring relationships among communication, sexual satisfaction, and marital satisfaction," Journal of Sex and Marital Therapy, vol. 31, no. 5, pp. 409-424, 2005.

[8] C. A. Wenner, Is sex important to marital satisfaction or is marital satisfaction important to sex? Top-down and bottom-up processing in the bedroom [M.S. thesis], University of Tennessee, 2010, http://trace.tennessee.edu/utk_gradthes/672.

[9] H.-C. Yeh, F. O. Lorenz, K. A. S. Wickrama, R. D. Conger, and G. H. Elder Jr., "Relationships among sexual satisfaction, marital quality, and marital instability at midlife," Journal of Family Psychology, vol. 20, no. 2, pp. 339-343, 2006.

[10] J. K. McNulty and T. D. Fisher, "Gender differences in response to sexual expectancies and changes in sexual frequency: a shortterm longitudinal study of sexual satisfaction in newly married couples," Archives of Sexual Behavior, vol. 37, no. 2, pp. 229-240, 2008.

[11] B. K. Ashdown, J. Hackathorn, and E. M. Clark, "In and out of the bedroom: sexual satisfaction in the marital relationship," Journal of Integrated Social Sciences, vol. 2, no. 1, pp. 40-57, 2011.

[12] M. Z. Nezhad and A. M. Goodarzi, "Sexuality, intimacy, and marital satisfaction in Iranian first-time parents," Journal of Sex and Marital Therapy, vol. 37, no. 2, pp. 77-88, 2011.

[13] N. Moshtagh, N. Teimourpour, and A. Pourshanbaz, "The relationship between attachment styles, marital satisfaction and sex guilt with sexual desire in Iranian women," Iranian Journal of Clinical Psychology, vol. 1, pp. 18-28, 2012.

[14] "The effect of sexual skills training on marital satisfaction," Procedia-Social and Behavioral Sciences, vol. 30, pp. 2581-2585, 2011.

[15] S. Marandi, F. Azizi, B. Larijani, and H. R. Samshidi, Health in Islamic Republic of Iran, Ministry of Health and Medical Education, Unicef, Tehran, Iran, 1st edition, 2000.

[16] R. F. Stahmann, "Premarital counselling: a focus for family therapy," Journal of Family Therapy, vol. 22, no. 1, pp. 104-116, 2000.

[17] B. K. Vural and A. B. Temel, "Effectiveness of premarital sexual counselling program on sexual satisfaction of recently married couples," Sexual Health, vol. 6, no. 3, pp. 222-232, 2009.

[18] A. Montazeri, M. Vahdaninia, S. J. Mousavi, and S. Omidvari, "The Iranian version of 12-item short form health survey (SF12): factor stucture, internal consistency and construct validity," BMC Public Health, vol. 9, article 341, 2009.

[19] A. Montazeri, A. M. Harirchi, M. Shariati, G. Garmaroudi, M. Ebadi, and A. Fateh, "The 12-item general health questionnaire (GHQ-12): translation and validation study of the Iranian version," Health and Quality of Life Outcomes, vol. 1, article 66, 2003.

[20] N. Bahrami, N. Sattarzadeh, F. Ranjbar Koochaksariie, and M. Ghojazadeh, "Comparing depression and sexual satisfaction in fertile and infertile couples," Journal of Reproduction \& Infertility, vol. 8, pp. 52-59, 2007.

[21] B. J. Fowers and D. H. Olson, "ENRICH marital satisfaction scale: a brief research and clinical tool," Journal of Family Psychology, vol. 7, no. 2, pp. 176-185, 1993.

[22] M. Mirzadeh and R. Fallahchay, "The relationship between forgiveness and marital satisfaction," Journal of Life Sciences and Biomedicine, vol. 2, no. 6, pp. 278-282, 2012.

[23] J. S. Carroll and W. J. Doherty, "Evaluating the effectiveness of premarital prevention programs: a meta-analytic review of outcome research," Family Relations, vol. 52, no. 2, pp. 105-118, 2003.

[24] G. Trudel, "Sexuality and marital life: results of a survey," Journal of Sex and Marital Therapy, vol. 28, no. 3, pp. 229-249, 2002.

[25] A. Rostami, M. Ghazinour, L. Nygren, M. Nojumi, and J. Richter, "Health-related quality of life, marital satisfaction, and social support in medical staff in Iran," Applied Research in Quality of Life, vol. 8, pp. 385-402, 2013. 


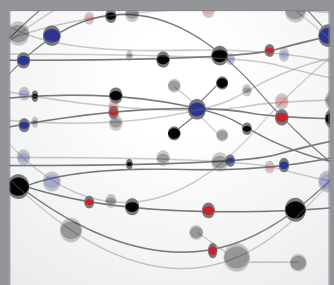

The Scientific World Journal
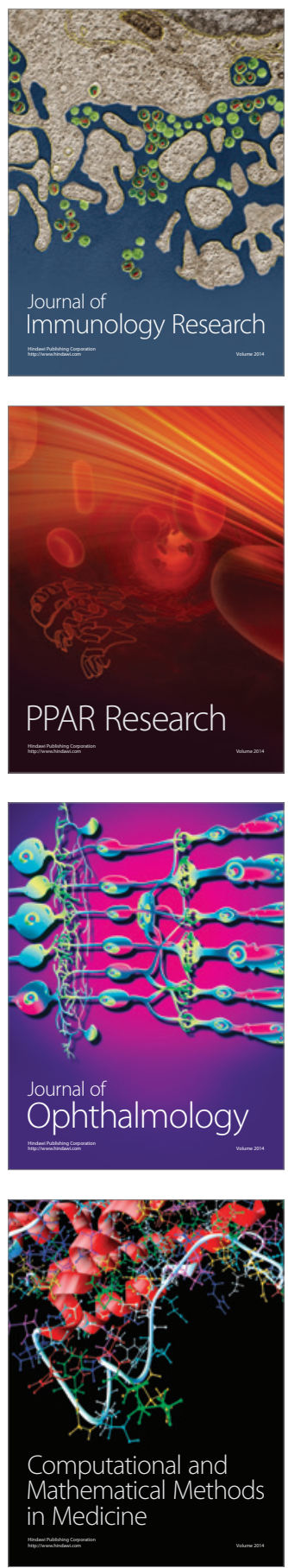

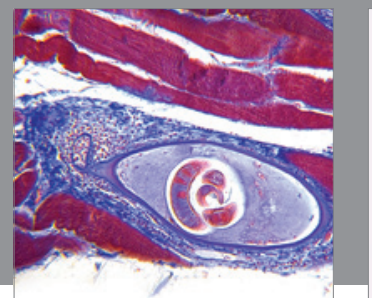

Gastroenterology

Research and Practice
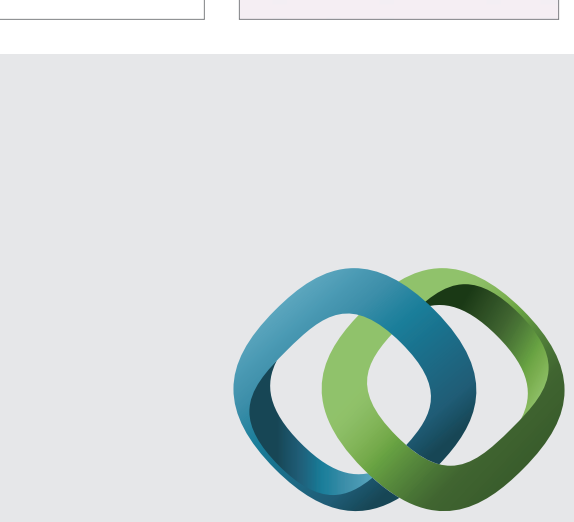

\section{Hindawi}

Submit your manuscripts at

http://www.hindawi.com
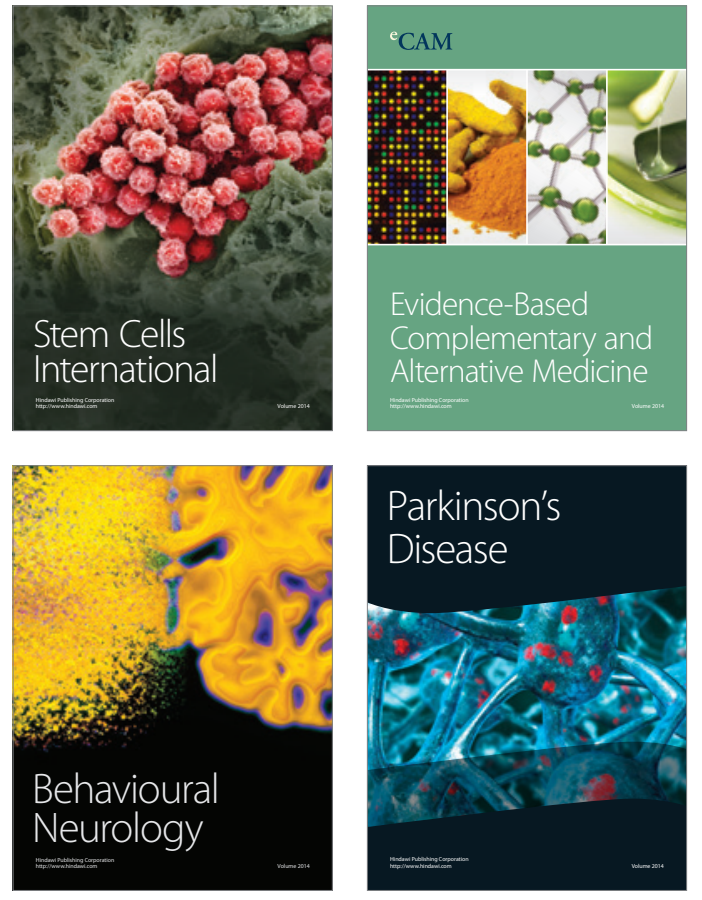
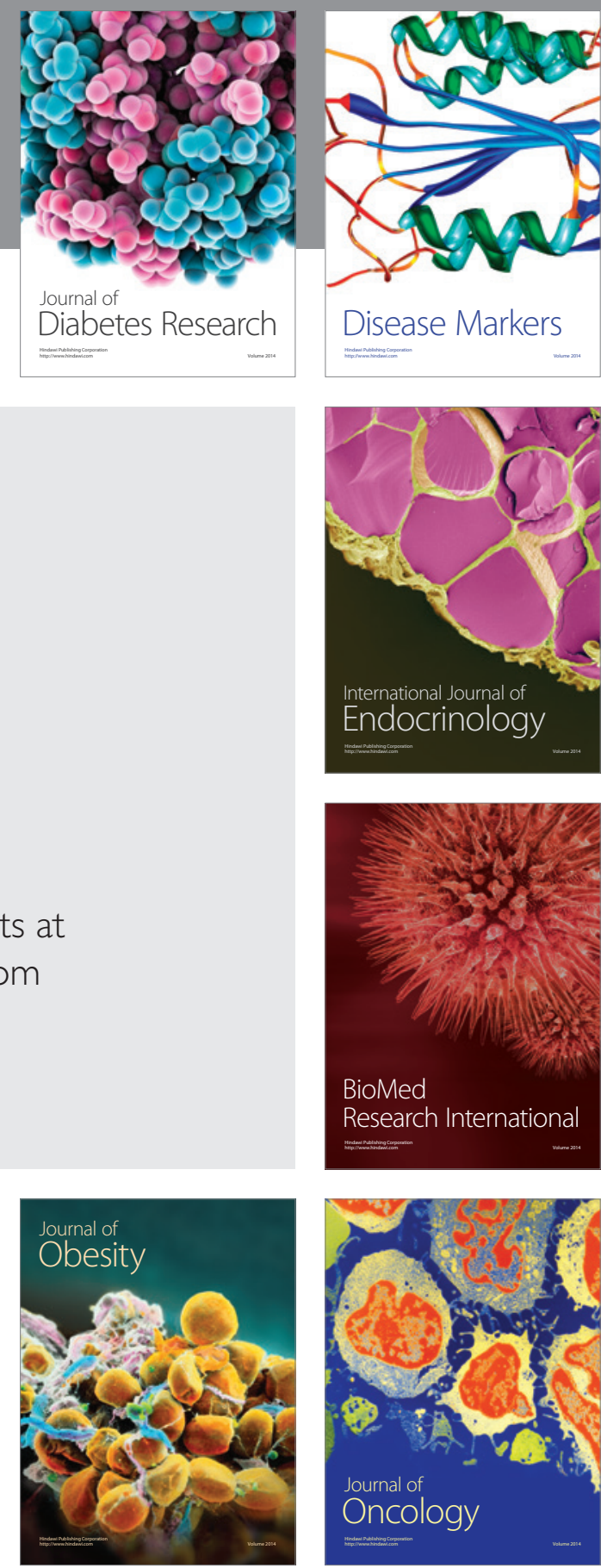

Disease Markers
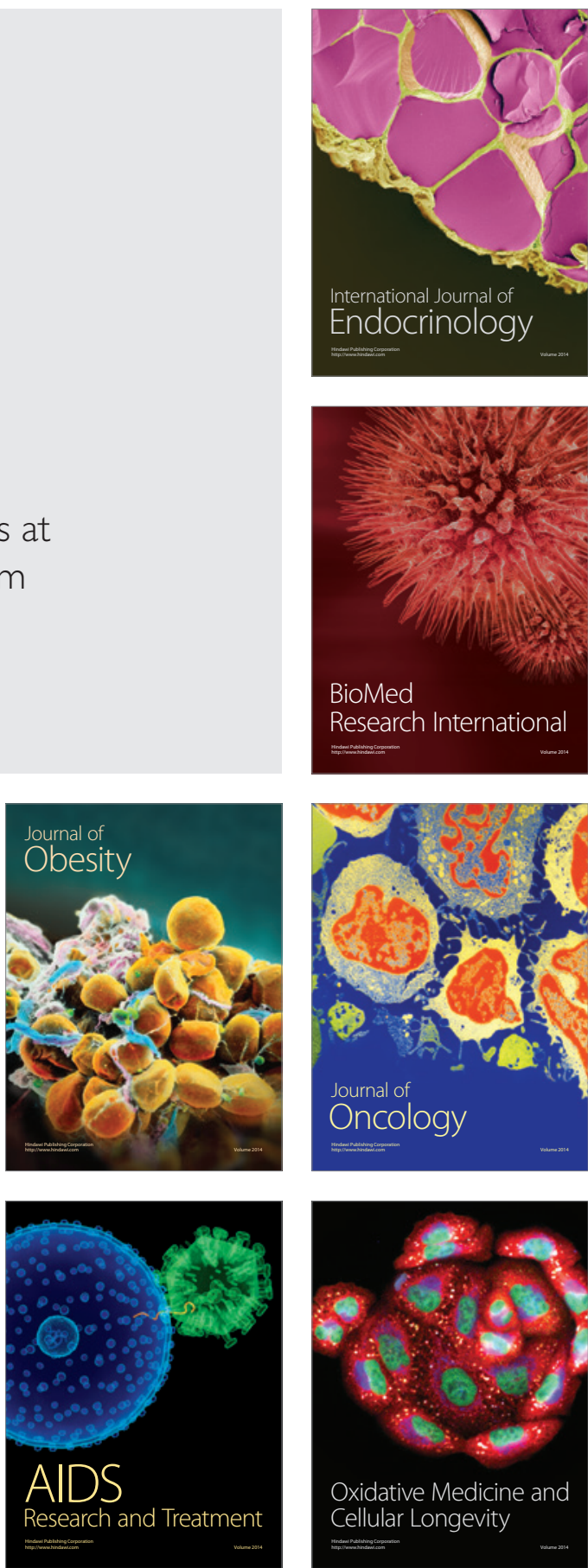